\title{
Non-invasive monitoring of avian embryo heart rate
}

\author{
Evgenii Aleksandrovich Andrianov (iD. Alexander Nikolaevich Sudakov (DD. \\ Aleksei Aleksandrovich Andrianov (iD. Nikolay Yakovlevich Skolznev iD
}

EA Andrianov - AN Sudakov (Corresponding author) - AA Andrianov

Voronezh State Agrarian University named after Emperor Peter the Great, ul. Michurina 1, Voronezh, 394087, Russian

Federation.

email: ansudak@gmail.com

\section{Received: January 14, 2019 - Revised: February 04, 2019 - Accepted: February 22, 2019}

\begin{abstract}
Non-invasive monitoring of the heart rate allows measuring the condition of the chick embryo in the incubation process without negative consequences for the future chick. The optical method for registering heartbeats is available to a wide range of incubation specialists and enables the registration of the heart rhythm of a developing embryo directly in an incubator. Long-term monitoring of the heart rate allows determining temperature regime compliance of incubation and adjusting the egg cooling duration. The experimental results demonstrate the availability of the method to a wide range of specialists. The results found in the present study reveal the possibilities of monitoring the heart rhythm of the developing chick embryo. A technique for spectral analysis of the waveforms of the embryo's heart activity is proposed.
\end{abstract}

Keywords: embryogenesis, incubation temperature, pulse

\section{Introduction}

The most important parameter influencing the embryo development during artificial incubation is temperature. However, it should be borne in mind that the true embryo temperature is important, which may differ from the air temperature inside the incubator chamber. Because endogenous heat production begins in the embryogenesis middle (Mueller et al 2015), the embryo temperature exceeds the air temperature in the incubator and, despite the accurate maintenance in the incubator chamber temperature, embryos often die from overheating (Tzschentke and Janke 2006). The easiest way to determine the true embryo temperature is an invasive measurement, in which the thermometer is inserted inside the egg, however, this measurement type leads to the embryo death. Some researchers think that measuring the eggshell temperature allows determining the embryo temperature with an accuracy of $\pm 0.3{ }^{\circ} \mathrm{C}$ (Hulet et al 2007). Some hatching eggs manufacturers recommend periodically

\section{NY Skolznev}

Voronezh State University, 1, Universitetskaya Square, Voronezh, 394006, Russian Federation.

conducting eggshell temperature measurement and the temperature adjustment inside the incubator chamber based on these data.

When modern incubation regimes are used, which presuppose egg periodic cooling, it is especially important to control the embryo temperature. However, due to the egg has thermal inertness during cooling periods and subsequent heating, the measurement of the eggshell temperature may not inform about the true embryo temperature.

A viable solution to control the embryo temperature is the use of indirect methods of monitoring the state of the embryo (Sudakov and Andrianov 2018). In invasive experiments to study the temperature effects on the state of the embryo was detected change in the heart rate due to temperature effects on the egg (Bogue 1932). Modern research methods allow controlling the heart rate without damaging the eggshell and confirm the temperature effects on the embryo through changes in its pulse (Mortola et al 2010; Tazawa 1985).

There are several methods for non-invasive control of the embryo's heart rate (HR). Acoustographic studies record the acoustic vibrations created by the heart. Other studies recording the egg's vibrations caused by the blood moving through the arteries (Tazawa 2005). The optical method measures the change in the light transmittance of an egg due to a change in the veins diameter based on blood flow (Lierz et al 2006).

To date, avian embryo heart rate studies are carried out in specialized laboratories and are not used by incubatory specialists in poultry breeding centers. Our idea was to study the possibility of using heart rate monitoring in poultry breeding centers.

\section{Materials and Methods}

We chose the optical method of heart rate control since the controlling egg vibration method is very sensitive to 
external interference and requires special changes in the incubator design and the acoustographic method. This would make it difficult in the late period of embryogenesis due to the influence of the embryo's motor activity on recorded sound vibrations.

The experiment used 30 pieces of a fertilized egg of domestic hens (Gallus gallus), which was obtained from the parent flock kept in the laboratory. The incubation was carried out in an incubator R-com 50 pro at a temperature of $37.8 \pm 0.2$ ${ }^{\circ} \mathrm{C}$ and humidity of $60 \pm 5 \%$. The eggs were rotated $\left(90^{\circ}\right)$ in automatic mode once every 60 minutes. The experiment was performed on the 15th day of incubation. The heart rate monitoring duration for each egg was 2 hours. Heart rate monitoring started 30 minutes before cooling. The cooling imitation, taken with a thermo-contrast incubation mode, was carried out by turning off the heating element of the incubator with its lid open for 10 minutes. Heart rate control was obtained using a sensor consisting of four IR LEDs and a photodiode, which was placed in the incubator chamber under the egg and connected to the amplifier with shielded wire. The amplified signal was plugged in an ordinary PLM 5-2 USB external sound card connected to a personal computer. Processing of the received signal was carried out in the program Adobe Audition 1.5. The air temperature control and the eggshell temperature was carried out using two thermistors connected to a personal computer through an analog-to-digital converter based on a microcontroller. The register and processing of temperature data were carried out in the Microsoft Excel program. Analog and digital graphics synchronization were carried out by overlaying images in the program Adobe Photoshop CS6 x32.

All manipulations with the eggs containing live embryos complied with the requirements of the legislation of the Russian Federation. After the experiment was completed, the incubation of the eggs was continued, and all the chicks received after its completion was returned to the farm.

\section{Results and Discussion}

As a result of the experiment on recording the embryo's heart rate in the incubator chamber, oscillograms of the heart rate during two hours were obtained are shown in Figure 1.

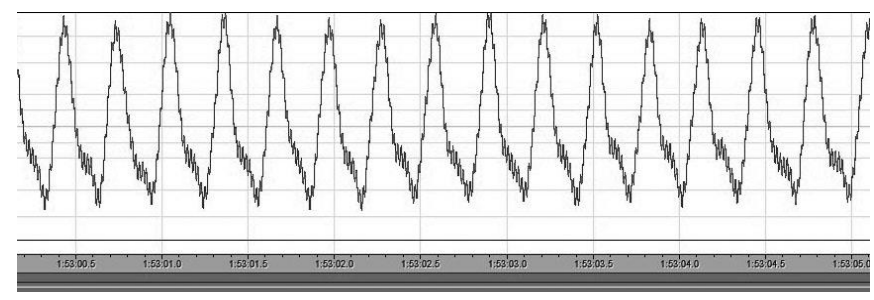

Figure 1 A fragment of the timeline and oscillograms of the chick embryo pulse on the 15th day of incubation.

$$
H R(b p m)=\frac{60 \times n}{t}
$$

where $n$ is the number of heartbeats during the time $(t, \mathrm{sec})$. In this case, the heart rate is 187 beats/minute.

In order to verify the recorded compliance of the heart rate values with the results obtained by other studies, we carried out of the embryo's heart rate calculation for all two hours that the experiment continued. The first result occurred in the sixth minute, then - every 10 minutes. According to the results of the experiment, Table 1 was compiled, which also included temperature sensors readings of eggshell temperature and air temperature in the incubator chamber.

Table 1 Temperature and heart rate in the experiment on cooling eggs on the 15 th day of incubation every 10 minutes.

\begin{tabular}{lllllllllllll}
\hline Time $(\mathrm{min})$ & 6 & 16 & 26 & 36 & 46 & 56 & 66 & 76 & 86 & 96 & 106 & 116 \\
\hline Air temperature $\left({ }^{\circ} \mathrm{C}\right)$ & 37.3 & 37.3 & 37.3 & 22.1 & 23.3 & 36.9 & 37.3 & 37.3 & 37.3 & 37.1 & 37.3 & 37.3 \\
Eggshell temperature $\left({ }^{\circ} \mathrm{C}\right)$ & 37.5 & 37.5 & 37.7 & 31.8 & 29.1 & 35.4 & 36.1 & 36.6 & 37.3 & 37.5 & 37.7 & 37.7 \\
Heart rate (beats.min $\left.{ }^{-1}\right)$ & 218 & 231 & 233 & 199 & 157 & 137 & 198 & 218 & 208 & 208 & 188 & 212 \\
\hline
\end{tabular}

Our results demonstrate a dependence relationship between the embryo heart rate and egg temperature. These results are confirmed by the Lierz et al (2006), which studied the effect of temperature on the turkey embryo heart rate using a digital egg monitor using infrared light. The data also correlate with studies based on recording acoustic oscillations of cardiac activity (Andrewartha et al 2011).

Simultaneously, the heart rate was determined using the method of counting the number of heartbeats for a certain time period does not allow for monitoring the embryo state in real time and requires considerable time from the experimenter.

The presented table (Table 1) is of greater interest to scientists, however, it is not informative for hatchery workers who need to observe the changes dynamics in the embryo heart rate during the cooling eggs when using the thermocontrast incubation mode. Significantly more informative is the graph (Figure 2), based on the results of Table 1.

The results showed in Figure 2 demonstrates a correlation between embryo heart rate and egg temperature. However, it is possible to note the decline in the heart rate, 
which continues after cooling is stopped. Due to the egg content has thermal inertness, even after the 46th minute of the experiment, after the resumption of the incubator heaters, the eggshell temperature increased, the heart rate continued to decrease due to the embryo inertial temperature.

Considering that one of the ways to control the embryo temperature during industrial incubation is to control the eggshell temperature (Boerjan 2005; Lourens et al 2005). The correlation between the heart rate and the eggshell temperature was 0.58 , which is significantly lower than the relationship of the eggshell temperature with the air temperature in the incubator chamber (0.93).

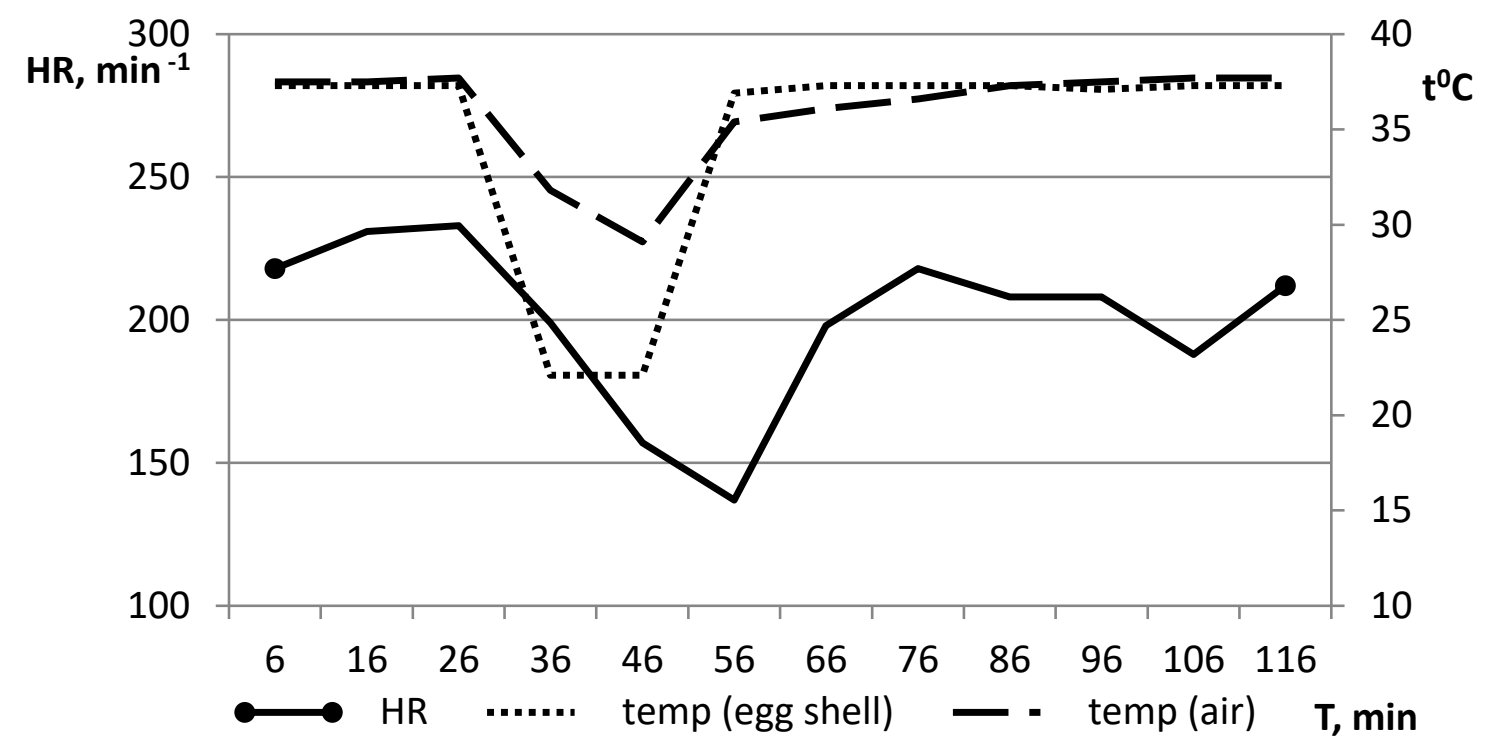

Figure 2 Changes in the heart rate, eggshell temperature and air temperature in the incubator for two hours during the experiment to eggs cooling for 10 minutes.

The low frequency of time sampling of the presented materials may distort the true information. The decrease in heart rate in the 106th minute of the experiment is not supported by data on temperature changes. As mentioned above, obtaining more frequent heart rate indicators using the calculation method takes considerable time. In this case, one can only suggest what caused heart rate changes, an error in the calculations, or, as observed in other studies, natural fluctuations in heart rate during the late periods of embryogenesis.

As a search result for a simple method of observing and recording the heart rate of an avian embryo in real time, we carried out a spectral analysis of the frequency response of the waveform. Despite a fast decrease in the accuracy of the obtained values, this method allows real-time observation in the embryo's heart rate changes (Figure 3).

The spectrogram (Figure 3) demonstrates a delay in heart rate increase after egg heating resumption. The embryo heart rate fluctuations noted on the spectrogram confirm the observations of other researchers about the presence of shortterm changes in the heart rate during the late periods of embryogenesis. It should also be noted that, despite the similar characteristics of heart rate graphs, obtained by the calculation method and the spectral analysis method, this latter allowed to avoid errors generated in the event of a calculation point at the peak value of short-term in heart rate changes.

Despite the registration of the embryo's motor activity in the present study was not supposed, the dark blue parts of the spectrogram indicate a decrease in the embryo's motor activity.

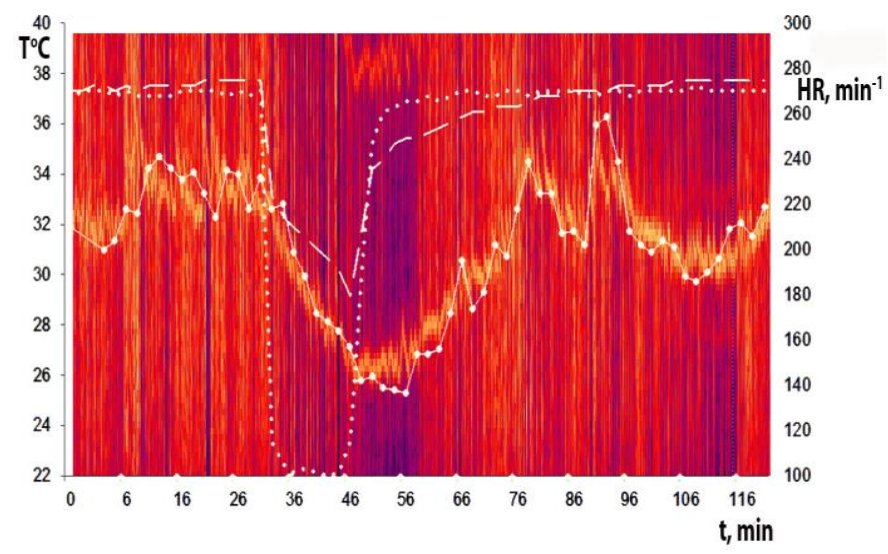

Figure 3 Frequency response spectrogram of the signal from the device for heart rate optical recording (orange band), obtained in the experiment on cooling eggs on the 15th day of incubation for 15 minutes. For more information, superimposed graphs of temperature and calculated values of heart rate are provided. 


\section{Conclusions}

We conclude that the eggshell temperature does not correspond to the true embryo temperature during temperature change periods in the incubator chamber. Spectral analysis of the in the response of signal frequency obtained by the optical recording of the heart rate of an avian embryo makes it possible to observe, in real time, the change dynamics in the embryo heart rhythm due to egg cooling. There was a slight decrease in the accuracy of the instantaneous heart rate; however, this method allows observing in detail the embryo cardiac activity framework. The presented method allows a wide range of incubation specialists to obtain a new tool for analyzing their work results.

\section{References}

Andrewartha, SJ, Tazawa H, Burggren WW (2011) Embryonic control of heart rate: examining developmental patterns and temperature and oxygenation influences using embryonic avian models. Respir. Physiol. Neurobiol. 178:84-96.

Bogue JY (1932) The heart rate of the developing chick. J. Exp. Biol 9:351-358.

Boerjan M (2005) Genetic progress inspires changes in incubator technology. Pas Reform Hatchery Technologies. Bovendorpsstraat 11, 7038 CH, P.O. Box 2, 7038 ZG Zeddam, The Netherlands.

Hulet R, Gladys G, Hill D, Meijerhof R, El-Shiekh T (2007) Influence of egg shell embryonic incubation temperature and broiler breeder flock age on posthatch growth performance and carcass characteristics. Poultry Science 86:408-412.
Lierz M, Gooss M, Hafez O, Hafez M (2006) Noninvasive heart rate measurement using a digital egg monitor in chicken and Turkey embryos. Journal of Avian Medicine and Surgery. 20:3.

Lourens A, Van den Brand H, Meijerhof R, Kemp B (2005) Effect of eggshell temperature during incubation on embryo development, hatchability and post-hatch development. Poultry Science 84:914920 .

Mortola J, Wills K, Trippenbach T, Al Awam K, (2010) Interactive effects of temperature and hypoxia on heart rate and oxygen consumption of the 3-day old chicken embryo. Comp. Biochem. Physiol. 155:301-308.

Mueller C, Burggren W, Tazawa H (2015) The Physiology of the Avian Embryo. Developmental and Integrative Biology. DOI: 10.1016/B978-0-12-407160-5.00032-4

Sudakov A, Andrianov E (2018) Real-time bird embryo monitoring. Biotechnologies and innovations in agribusiness: Mater. int. scientific-practical conf. - Belgorod: Belgorod. state agrarian University Press. 2:80-84.

Tazawa H (2005) Cardiac rhythms in avian embryos and hatchlings. Avian Poult. Biol. Rev. 16:123-150.

Tazawa H, Nakagawa S (1985) Response of egg temperature, heart rate and blood pressure in the chick embryo to hypothermal stress. J. Comp. Physiol. 155:195-200.

Tzschentke B, Janke O (2006) Development in heat production and em-bryonic temperature. Lincoln. 3:33. 